\title{
Ambientes web para 0 ensino de superfícies topográficas em realidade aumentada e realidade virtual
}

WEB ENVIRONMENTS FOR TEACHING TOPOGRAPHIC SURFACES IN AUGMENTED REALITY AND VIRTUAL REALITY

\author{
Paulo Henrique Siqueira \\ Departamento de Expressão Gráfica, Universidade Federal do Paraná, Curitiba, PR, Brall \\ E-MALL: PAULOHS@UFPR.BR
}

\begin{abstract}
This paper shows the use of web resources for the creation of Virtual Reality (VR) and Augmented Reality (AR) environments to teaching of topographic surface concepts. Surface virtual mockups built with Sketchup software features include graphical representations of level contours, plan sections, and embankment slopes. The terrains textures used are satellite photos available by the Digital Globe platform and contribute to improve the visualization of studied concepts. Developed environments enable students to view AR surfaces through their webcam devices, such as smartphones, tablets, or notebooks, from a variety of points of view. Each topographic surface has a link to its respective representation in VR, which allows its manipulation and detailed study of each concept. The environments presented in this work can be used in disciplines of Topography, Geography and Descriptive Geometry.
\end{abstract}

Resumo: Este artigo mostra o uso de recursos web para a criação de ambientes de Realidade Virtual (RV) e Realidade Aumentada (RA) para o ensino de conceitos sobre superfícies topográficas. As maquetes virtuais das superfícies construídas com recursos do software Sketchup incluem as representações gráficas de curvas de nível, seções planas e taludes de aterros. As texturas dos terrenos utilizados são imagens de satélite disponibilizadas pela plataforma Digital Globe e contribuem para melhorar a visualização dos conceitos estudados. Os ambientes desenvolvidos possibilitam que os alunos visualizem as superfícies em RA por meio de seus dispositivos com webcam, tais como smartphones, tablets ou notebooks, observando-as em diversos pontos de vista. Cada superfície topográfica modelada possui um link para sua respectiva representação em RV, que possibilita sua manipulação e estudo detalhado de cada conceito. Os ambientes apresentados neste trabalho podem ser usados em disciplinas de Topografia, Geografia e Projeções Cotadas.
Citation/Citação: Siqueira, P. H. (2020). Ambientes web para 0 ensino de superfícies topográficas em realidade aumentada e realidade virtual. Terræ Didatica, 16, 1-9, e20001. doi: 10.20396/td.v16i0.8655900.

Keywords: Augmented Reality, Virtual Reality, Topographic surfaces, Virtual Mockups.

Palavras-chave: Realidade aumentada, Realidade virtual, Superfícies topográficas, Maquetes virtuais.

\section{Manuscript/Manuscrito:}

Received/Recebido: 13/07/2019

Revised/Corrigido: 18/09/2019

Accepted/Aceito: 19/01/2020

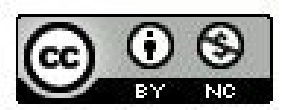

\section{Introdução}

O uso de recursos auxiliares permite a melhor compreensão dos alunos em disciplinas que envolvem conceitos tridimensionais. A manipulação de objetos auxilia na compreensão dos alunos, tornando mais fácil a assimilação dos conceitos teóricos das disciplinas. A construção de modelos e maquetes físicas podem ser feitas, hoje em dia, com o uso de impressoras 3D para aulas de Geografia (Sena \& Carmo, 2018), Biologia (McMenamin et al., 2014), Geometria (Huleihil, 2017) e outras disciplinas que envolvem representações em 3D. O desenvolvimento de ambientes ou aplicativos web para o ensino também ajuda a visualização de conceitos de Biologia (Attardi \& Rogers, 2015) e Geometria (Cantos et al., 2016).

$\mathrm{O}$ uso de modelagem com tecnologias virtuais é uma das alternativas para auxiliar o ensino em disciplinas que envolvem conceitos em 3D. A Realidade Virtual (RV) serve para criar um ambiente imersivo com manipulação dos objetos por meio de controles e óculos imersivos (Moro et al., 2017). Ambientes em RV auxiliam a visualização de fenômenos físicos ou biológicos, simulações de treinamentos, visualização de superfícies de planetas, simulações de construções, jogos educacionais e outras áreas da educação.

A Realidade Aumentada (RA) utiliza um dispositivo com câmera para inserir objetos junto com o ambiente da imagem da câmera, criando camadas virtuais de objetos 3D e texto sobre a imagem da câmera em tempo real (Kirner \& Kirner, 2011). Trabalhos recentes mostram aplicações interessantes de RA para auxílio no ensino de várias áreas, tais como Geometria (González, 2017), Engenharia (Cerra et al., 2018), Química (Irwansyah et al., 
2018) e Arquitetura (Abdullah et al., 2017). As contribuições do uso de RA na área da educação demonstram que se trata de uma poderosa ferramenta para uso em sala de aula, pois permite várias formas de interações visuais no aprendizado de diversas disciplinas (Akçayir \& Akçayir, 2017).

Neste trabalho são apresentados os elementos necessários para a construção de um ambiente web que utiliza as tecnologias de RV e RA para representar maquetes virtuais para o ensino de superfícies topográficas. As maquetes são construídas no software Sketchup com os recursos de texturas e modelagem disponíveis da plataforma Digital Globe. Os desenhos de seções planas, plataformas, taludes de aterros e curvas de nível são feitos no Sketchup com a inserção nos ambientes programados de RA e RV, possibilitando um estudo aprofundado das feições dos terrenos e das principais formas de relevo: vales, colinas, serras, montanhas, lagos, planaltos e planícies.

A ideia proposta neste trabalho é de utilizar uma página em HTML para a RA, com links para as páginas desenvolvidas em RV. Na página em $\mathrm{RA}$, os alunos visualizam as maquetes por meio de diversos pontos de vista e acessam os sites de RV para manipularem as representações das superfícies topográficas com dispositivos móveis, computadores ou podem até imergirem na cena com óculos de RV.

Os comandos usados na construção dos ambientes propostos em RA e RV são intuitivos, e exigem apenas conhecimentos básicos de HTML. Trata-se de um recurso didático de programação simples, que possibilita aplicações em sala de aula sem dificuldades apontadas no uso de algumas tecnologias de RA, conforme mostra o trabalho de Munoz-Cristóbal et al. (2018).

\section{Ensino de superfícies topográficas}

O estudo de superfícies topográficas pode ser aprimorado com o auxílio das tecnologias virtuais. A construção de elementos em 2D das representações gráficas de terrenos pode ser complementada por meio de visualizações $3 \mathrm{D}$, geradas por meio de diversas técnicas disponíveis.

De acordo com Andrade et al. (2018), a tecnologia de Caixa de Areia (Sandbox) tem sido bastante utilizada no ensino de formas de relevo na disciplina de Geografia. O sistema faz a leitura das camadas de areia, criando as curvas de nível por intermédio de cores definidas pelas técnicas de hipsométricas e batimétricas, fazendo com que os alunos tenham a percepção do formato dos terrenos. O trabalho de Santos et al. (2018) mostra o uso da ferramenta Sandbox para visualizações de regiões de Sergipe e aplicações de estudos de declividades da disciplina de Topografia para o curso de Engenharia Civil.

O uso dos conceitos de Geometria Cotada para representação de um projeto simplificado de instalação de linhas de tensão é mostrado no trabalho de Santos et al. (2003). As informações iniciais apresentadas aos alunos das cartas topográficas são usadas para construção do modelo 3D, com todas as normas técnicas para a correta instalação de cada torre de transmissão.

O trabalho de Souza et al. (2016) mostra o uso da Realidade Aumentada na construção de um mapa do campus de Recife, da Universidade Federal de Pernambuco, com as informações cartográficas disponíveis. O modelo foi testado com alunos do curso de Engenharia Cartográfica, e a maioria considerou que o modelo em RA melhora a visualização em comparação com mapas tradicionais. O mapa em RA proposto pelos autores levou em conta apenas as feições do terreno e os formatos dos edifícios construídos no campus.

As maquetes físicas também são importantes para o ensino de superfícies topográficas. Os trabalhos de Becker \& Nunes (2012), Silva \& Ferreira (2008) e Sousa (2014) mostram as atividades para construir as maquetes físicas com os alunos para o auxílio no ensino de conceitos de relevos e curvas de nível. Os resultados mostrados por esses autores são excelentes, com a interação entre os alunos e professores, resultando na melhoria da compreensão dos conceitos de superfícies topográficas pelos alunos.

O trabalho de Carrera \& Asensio (2017) utiliza a Realidade Aumentada para visualizações das superfícies topográficas empregando-se o aplicativo AR-Media. Com as curvas de nível impressas de cada terreno, os autores mostram o funcionamento do aplicativo com as superfícies que aparecem sobre as curvas, complementando o ensino das formas de relevo.

O uso da Realidade Aumentada pode complementar a utilização dos materiais didáticos tradicionais no ensino dos conceitos de superfícies topográficas, pois os alunos podem interagir e visualizar os elementos dos terrenos de maneira mais efetiva e significativa. A Realidade Virtual pode auxiliar na interação dos alunos com as representações 3D dos terrenos, transformando o ensino dos conteúdos em uma espécie de aula de campo sobre relevos de variadas formas. 


\section{Modelagem das superfícies topográficas}

Os modelos de maquetes mostrados neste artigo foram construídos no software Sketchup (2019) com o uso do recurso disponível para superfícies terrestres da plataforma Digital Globe (2019). Os modelos são exportados para que sejam inseridos nos ambientes web programados em A-frame (2019) para uso tanto em Realidade Aumentada quanto em Realidade Virtual. Desta forma, os conceitos estáticos das formas de relevo dos terrenos podem ser visualizados da forma como são, tornando os conteúdos destas aulas mais realísticos e dinâmicos.

O primeiro passo para a construção do ambiente proposto neste artigo é a escolha de regiões que contém vales e montanhas interessantes para uso em sala de aula. A ferramenta de inserção de terrenos da Digital Globe cria janelas de visualização de tamanhos limitados a quadrados de lados iguais a 2 $\mathrm{km}$, cuja modelagem é feita apenas para a superfície terrestre, desconsiderando-se as alturas de construções e de vegetações. As texturas usadas nesta ferramenta são as imagens de satélite, ideais para que as superfícies modeladas fiquem mais próximas da realidade em RA e RV.

Após escolher a localização do terreno, a imagem do satélite da região é carregada no Sketchup, conforme mostra a Figura 1. Trata-se da vista superior do terreno, que neste exemplo é de uma região de vales de Jolster, na Noruega. Com a opção do Sketchup de mostrar o terreno, a superfície topográfica pode ser usada com as altitudes reais da região escolhida. A Figura 2 mostra a modelagem da região de Jolster em $3 \mathrm{D}$ com os recursos da plataforma Digital Globe.

A construção do conjunto de curvas de nível da região pode ser feita por meio de retângulos equidistantes em relação ao eixo $z$ (cotas ou altitudes), com lados paralelos aos eixos x e y e medidas um pouco maiores do que as laterais da superfície. Estes retângulos representam os planos horizontais de seção da superfície. O resultado das interseções destes planos equidistantes com a superfície é o conjunto de curvas de nível, conforme mostra a Figura 3. Outros elementos importantes que podem ser definidos no Sketchup são as cotas das curvas de nível e os planos de seção e de aterros. A vista superior de cada superfície pode ser usada para criação de materiais didáticos sobre as superfícies topográficas.

Se a região escolhida for maior do que a limitação de $2 \mathrm{~km}$ da janela de visualização do Digital

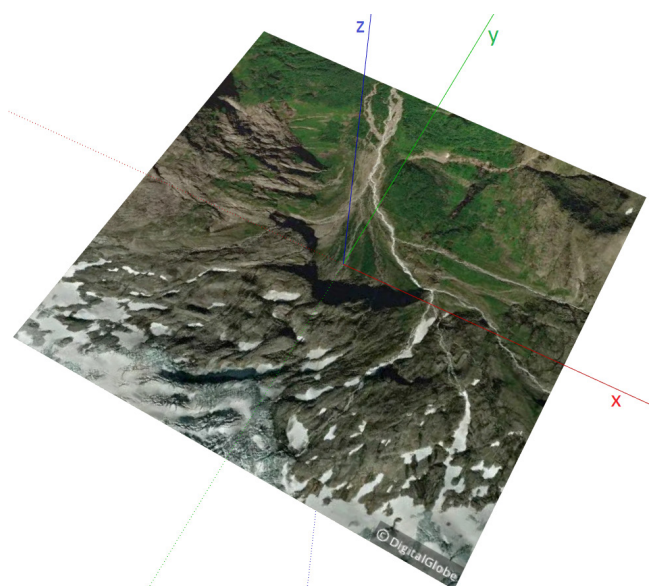

Figura 1. Imagem de satélite da região de vales de Jolster, Noruega. Fonte: 0 autor

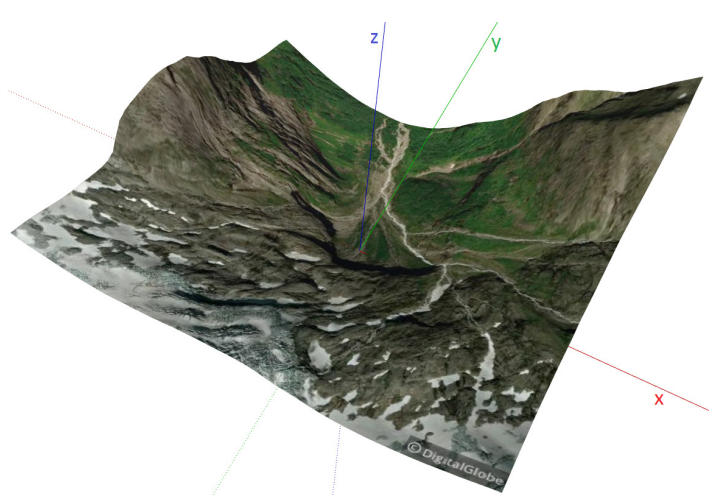

Figura 2. Modelo em 3D da região de vales de Jolster. Fonte: o autor

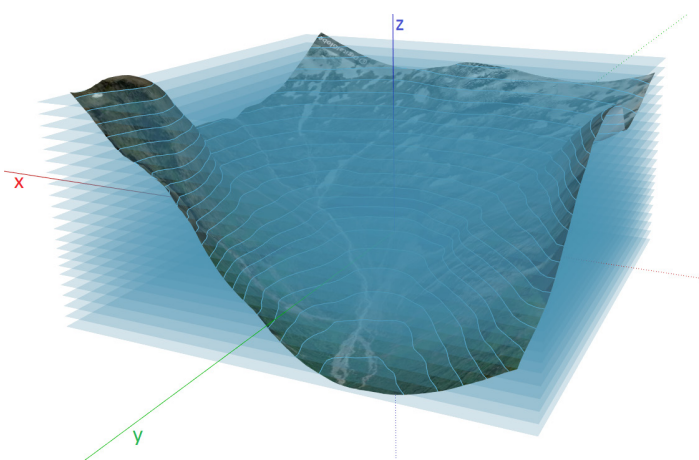

Figura 3. Construção das curvas de nível da região de Jolster. Fonte: o autor

Globe, podem ser criadas diversas janelas com as sub-regiões que cobrem a área escolhida, e cada imagem se encaixa com a imagem vizinha, como uma espécie de colcha de retalhos.

Para tornar ainda mais interessante o uso das tecnologias propostas neste trabalho, a região escolhida para uma determinada atividade pode ser turística, como o Monte Fuji no Japão, o Monte 
Everest que fica na fronteira entre a China e o Nepal, ou a Chapada Diamantina no Brasil. Para representar uma maquete de uma região do Grand Canyon, que fica no estado do Arizona dos Estados Unidos, são necessárias 40 janelas de visualização, que estão ilustradas na Figura 4 em 2D e na Figura 5 em 3D. Na maquete virtual uma grande região do Rio Colorado encontra-se representada, possibilitando a exploração de várias formas de relevo em sala de aula.

\section{Realidade Virtual}

Os modelos de superfícies topográficas mostrados na seção anterior foram inseridos nos ambientes de Realidade Virtual e de Realidade Aumentada por meio das bibliotecas do A-frame. Trata-se de um ambiente desenvolvido pela equipe de RV do Mozilla (Oliveira, 2018) que utiliza as funções da biblioteca Three.js do Java com tags do HTML puro, permitindo que toda a programação de RV ou RA seja feita em uma página da web, que segue a composição de tags com princípios de herança e hierarquia (A-Frame, 2019).

Os ambientes programados no A-frame têm suporte para quatro formatos de arquivos de maquetes. O primeiro é o obj (object file), formato muito utilizado que pode ser aberto em qualquer software 3D, cujos arquivos incluem as informações de coordenadas, mapas de texturas e faces poligonais. O segundo formato usado pelo A-frame é o collada (collaborative design activity), suportado em muitos softwares 3D e que utiliza padrão $x m l$ para carregar as informações de texturas, animações e iluminação. O terceiro formato que pode ser usado é o gltf (graphic library transmission format), que possui as mesmas informações do obj de forma mais compacta, obtendo-se carregamento mais rápido em aplicações web. A versão binária do $g l t f$, denominada $g l b$, é o quarto tipo de arquivo suportado pelo A-frame.

O formato glb é o mais indicado para aplicações web, pois tem, em média, 20\% do tamanho de um arquivo com extensão collada ou obj. Porém, o único formato que exporta linhas é o collada. Como a visualização de linhas é essencial para o estudo de retas e curvas de nível, os ambientes foram construídos com a modelagem dos terrenos em formato $\mathrm{glb} \mathrm{e}$ as linhas em formato collada.

As tags principais da modelagem de uma das superfícies topográficas estão ilustradas na Figura 6. $\mathrm{Na}$ tag de cabeçalho da página HTML está inserida

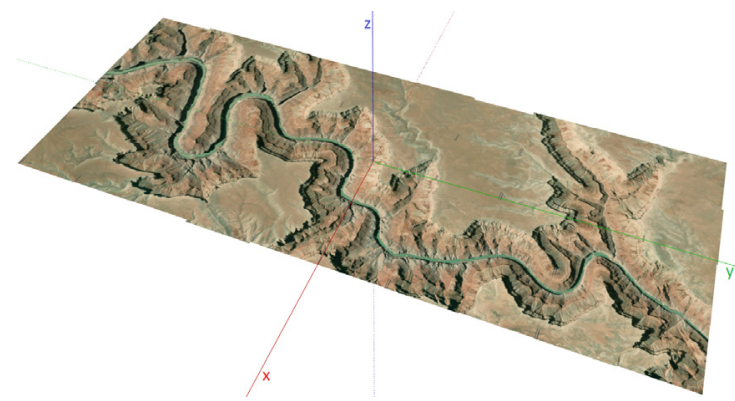

Figura 4. Janelas de visualização em 2D da maquete do Grand Canyon. Fonte: o autor

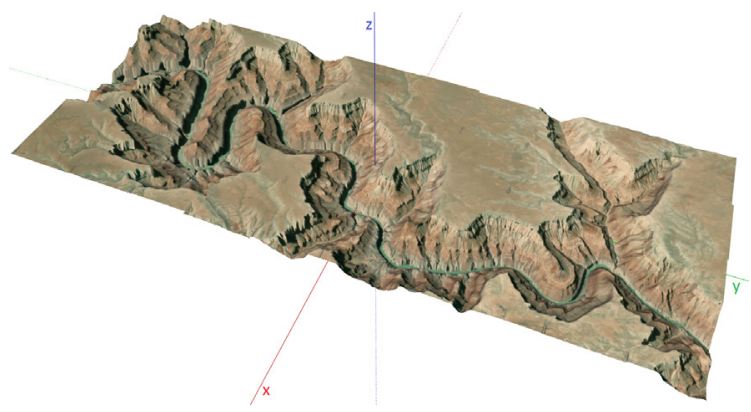

Figura 5. Representação em 3D da maquete do Grand Canyon. Fonte: 0 autor

a referência à biblioteca principal do A-frame entre as linhas 3 e 5 . Todas as referências de bibliotecas podem ser inseridas nesta tag de cabeçalho.

O corpo da página HTML contém a programação dos demais elementos da página. Nas linhas 7 e 8 da Figura 8 encontram-se as definições para interação do usuário com o mouse ou controle de RV e a câmera da cena com posição inicial nas coordenadas $x$ (direita/esquerda), $y$ (altura) e $z$ (profundidade). Os valores iniciais são: $x=0$ e $y$ $=0$, que centralizam a câmera na tela; e $z=6 \mathrm{~m}$ para afastar o observador da origem do sistema.

As tags que definem as texturas e o carregamento da maquete estão colocadas entre as linhas 9 e 13. O modelo está definido nas linhas 10 e 11, onde deve ser informado o caminho da pasta onde estão salvos os arquivos collada e gltf exportados do Sketchup. A imagem de fundo, com gradiente de tom de azul está definida na linha 12 e referenciada como imagem de fundo de $360^{\circ}$ da cena na tag $<a-s k y>$ da linha 14.

Entre as linhas 15 e 18 estão as tags que definem a posição e a escala da maquete. Para carregá-la adequadamente, a escala usada é de 0,003 nas três dimensões e a posição na origem do sistema. A Figura 7 mostra a maquete programada em RV com as tags mostradas na Figura 6. Trata-se da repre- 


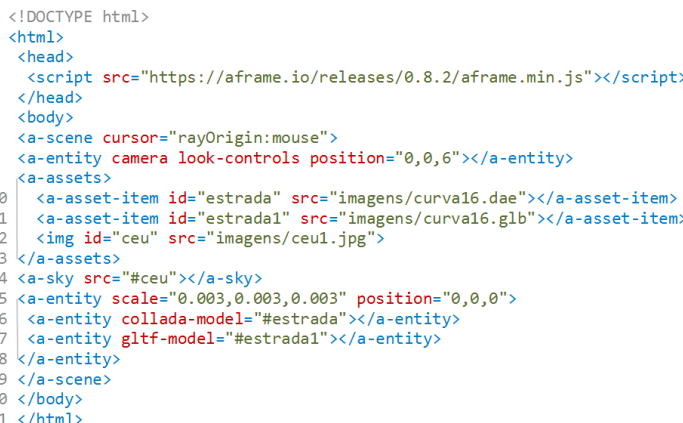

Figura 6. Código HTML em A-frame para apresentação em RV de uma maquete construída no Sketchup. Fonte: o autor

sentação gráfica usada para estudo de construção de estradas, com os taludes de aterro e corte do terreno. Com esta representação em RV, os alunos podem visualizar as construções que são feitas com as curvas de nível do terreno para definir os cortes e posições dos taludes em $2 \mathrm{D}$.

A interseção de uma superfície topográfica com o plano que define o talude de um corte ou de aterro pode ser feita por meio do encontro de cada curva de nível com a respectiva reta horizontal deste plano de mesma cota. Os alunos podem construir as curvas de corte e de aterro destes taludes em 2D e a complementação deste conceito pode ser feita por meio da visualização em 3D do que foi construído em RV.

Uma forma de interação com os elementos da cena é por meio da função de órbita (Hinrichs, 2019), que permite a movimentação da câmera em torno dos objetos da cena. Ao usar os óculos de RV, a movimentação da câmera com a função de órbita é automática. Nos computadores, tablets e smartphones, a movimentação da câmera em torno dos objetos pode ser feita por meio do mouse, teclado ou touch. Todas as maquetes usadas neste artigo têm esta função de órbita, para que os alunos possam movimentar a cena para encontrar os melhores pontos de vista para observar os elementos construídos.

Os projetos mostrados neste artigo são aplicados em disciplinas básicas de Topografia para projetos de Engenharia Civil, Engenharia Ambiental e Engenharia Florestal. Outros projetos que envolvem superfícies topográficas são de construções de seções planas para rampas. A Figura 8 mostra uma seção plana com as horizontais de $100 \mathrm{em} 100$ metros e as curvas de nível de 20 em 20 metros.

Por se tratar de uma página web, os usuários têm opções de visualização em tablets, computa-

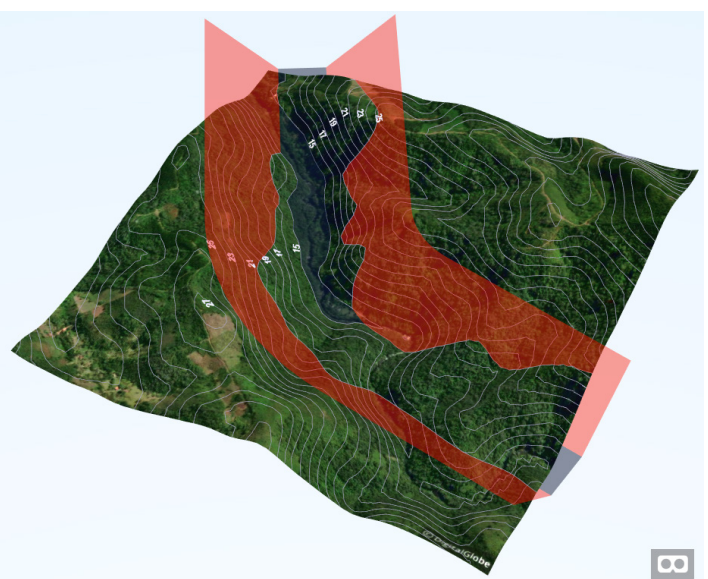

Figura 7. Representação em RV da maquete com cortes de terreno para construção de uma estrada. Fonte: o autor

dores, smartphones, além dos Oculus Rift, Oculus Vive, Daydream e gearVR. Os comandos de interações com as maquetes podem ser modelados na imersão da cena de RV para manipulações em órbita (Murphy, 2019) ou teleporte em locais da cena (Serrano, 2019). Com a função de teleporte, os alunos podem imergir em cada superfície com os óculos de RV, tornando o uso das maquetes em RV mais interessante: uma caminhada virtual do terreno representado.

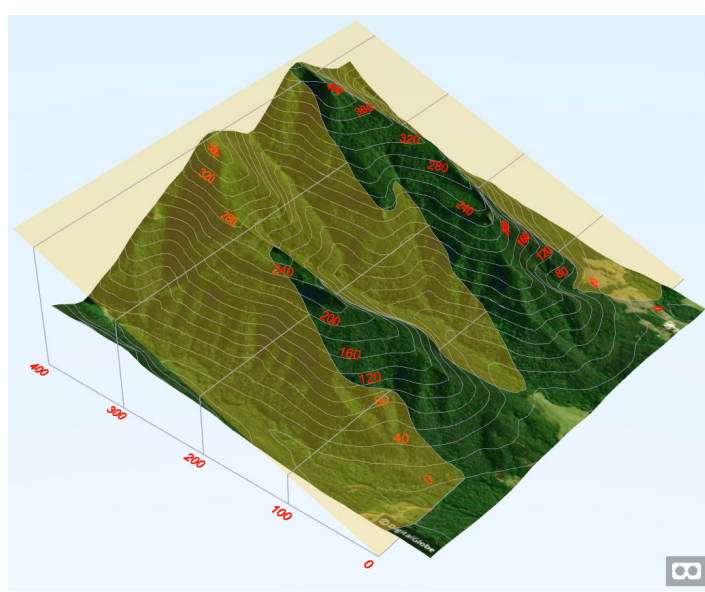

Figura 8. Representação em RV da maquete de uma seção plana de terreno. Fonte: o autor

\section{Realidade Aumentada}

Em um ambiente programado em Realidade Aumentada, os elementos modelados em Realidade Virtual podem ser misturados com objetos físicos mostrados por meio da câmera de um dispositivo. A programação da página web das maquetes em RA usa as mesmas tags estruturais mostradas na seção anterior, incluindo-se a tag referencial da visualiza- 


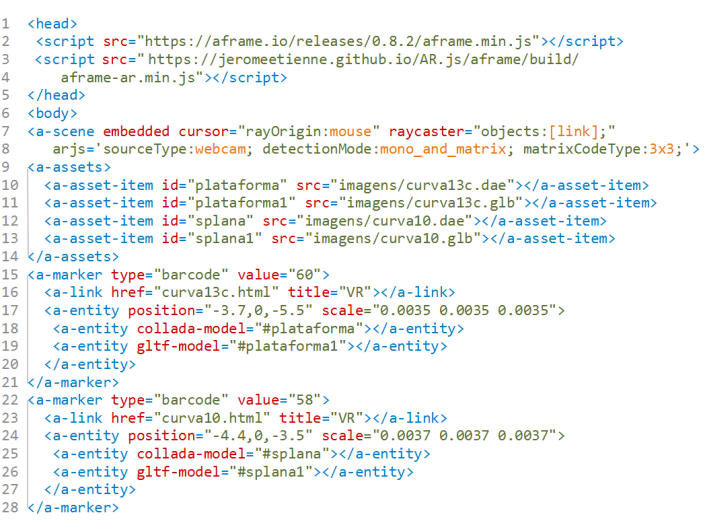

Figura 9. Marcadores usados no A-frame: hiro, kanji e $Q R$ codes \#58 e \#60. Fonte: o autor

ção em RA, desenvolvida por Etienne (2019), que deve ser inserida no cabeçalho da página junto com a tag referencial do A-frame.

A estrutura da página programada em HTML com as tags de duas maquetes está ilustrada na Figura 9. A tag da cena em RA tem a inclusão de propriedades de incorporação da imagem da webcam e captura de interação com mouse ou raios de controles em objetos com links (linhas 7 e 8). A cena em RA tem marcadores, que funcionam com códigos de bits 0 e 1 em forma matricial de imagens que são reconhecidas pela webcam (Ishii, 2010). Os marcadores funcionam como pontos de referência, onde podem ser estabelecidas posições específicas para os objetos virtuais que aparecem na imagem real da webcam.

Quando a imagem de um marcador for reconhecida na cena de RA, os elementos modelados em RV são ativados. Existem mais de 80 opções de marcadores programados na biblioteca desenvolvida por Etienne (2019), que são representados por tags que englobam os elementos programados em RV que são ativados. Os mais comuns são hiro, kanji e os QR codes, mostrados na Figura 10.
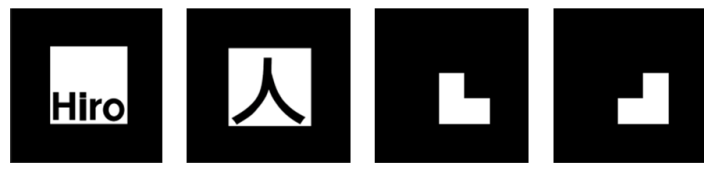

Figura 10. Código HTML em A-frame para apresentação em RA de duas maquetes construídas no Sketchup. Fonte: o autor

Com os marcadores impressos, os alunos podem acessar de seus dispositivos a página web. Assim, visualizam os marcadores programados; os respectivos objetos programados em RV aparecem nas telas dos dispositivos em RA. Os marcadores QR codes \#58 e \#60 foram usados para a visualização em RA das maquetes de seção plana e de plataforma com aterro e corte, respectivamente. A estrutura de tags da plataforma está entre as linhas 15 e 21 da Figura 9. Nas tags, a-entity serve para agrupar os elementos da programação em RV, posicioná-los sobre o marcador e usar escalas. As coordenadas mostradas na linha 17 servem para posicionar a maquete sobre a representação em 2D das curvas de nível. Desta forma, os alunos podem visualizar em 3D o desenho da seção plana construída em 2D que representa a vista superior da maquete.

A tag link, mostrada na linha 16 da Figura 9, cria a interação para acesso das páginas programadas em RV por meio de círculos azuis que aparecem sobre os marcadores. Entre as linhas 22 e 28 estão as tags da seção plana com o uso do $Q R$ code \#58. As referências para carregamento das maquetes estão inseridas na tag assets, entre as linhas 9 e 14 . As demais maquetes são programadas de maneira similar. A Figura 11 mostra as visualizações em RA da seção plana e da plataforma construídas pelos alunos por meio de Projeções Cotadas.

Com os marcadores impressos nos materiais didáticos das disciplinas, os alunos podem visualizar em 3D as construções que são feitas em 2D. Neste caso, as projeções ortogonais que representam a vista superior das curvas de nível de cada terreno estão representadas nas apostilas, e os alunos podem sobrepor os objetos em RA em seus desenhos para conferir os resultados ou somente visualizar para melhor compreensão dos conceitos estudados. Com o uso das tecnologias apresentadas neste artigo, os alunos conseguem materializar os conceitos de projeções e leituras de cartas topográficas de maneira mais eficiente e dinâmica.

Os modelos das maquetes utilizadas neste trabalho com formatos collada e glb são carregados rapidamente em qualquer dispositivo testado. A página desenvolvida em RA possui todos os links para visualizações dos elementos em RV mostrados neste trabalho e encontra-se disponível no endereço: https://paulohscwb. github.io/cotadas/superficies/. Ao entrar no site principal (https://paulohscwb.github.io/cotadas/ superficies/), o leitor pode conferir cada modelo 3D, por exemplo: 

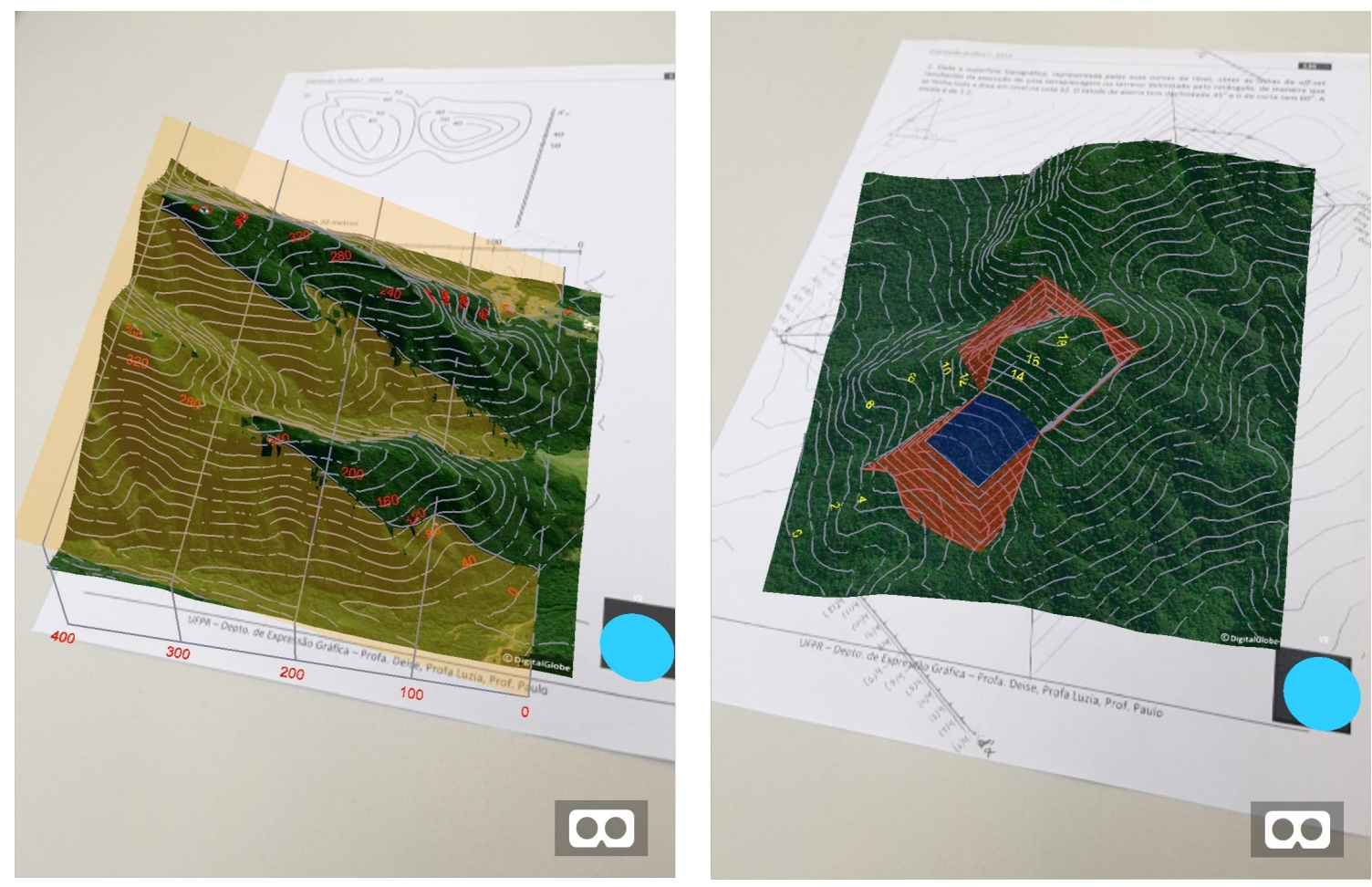

Figura 11. Visualização da seção plana e da plataforma com RA. Fonte: o autor

1. Modelo de curvas de nível: https://paulohscwb.github.io/cotadas/superficies/curva11. $\underline{\mathrm{html}}$

2. Modelo de perfil topográfico: https://paulohscwb.github.io/cotadas/superficies/curva3.html

3. Corte de terreno para estradas: https://paulohscwb.github.io/cotadas/superficies/curva15. $\underline{\mathrm{html}}$

Os materiais didáticos mostrados neste artigo foram usados em sala de aula com cinco turmas dos cursos de Engenharia Civil e Engenharia Florestal no primeiro semestre de 2019, totalizando 220 alunos. Os sites programados em RA e RV com as maquetes foram carregados sem falhas em todos os smartphones testados. Não foram reportados erros em carregamentos dos sites em notebooks e tablets. A única observação que merece destaque é de que os sites em RA costumam demorar um pouco mais para carregar totalmente, pois usam os recursos da webcam do dispositivo. Porém, os sites programados em RV têm carregamento praticamente imediato em todos os dispositivos testados, em qualquer sistema operacional.

As tecnologias mostradas em RA e RV das maquetes para a construção de plataformas e seções planas ajudaram na compreensão dos alunos nas leituras e construções que envolvem as superfícies topográficas. Muitos alunos conseguiam fazer as construções dos projetos de plataformas e seções planas de forma automática e mecânica, sem saber realmente quais eram as formas representadas em 3D. Com o uso da tecnologia proposta, a leitura da representação de um terreno por meio de curvas de nível ficou ao alcance de todos os alunos das disciplinas testadas neste trabalho.

\section{Considerações finais}

$\mathrm{O}$ artigo mostra um sistema baseado em web para visualização de superfícies topográficas em Realidade Virtual e Realidade Aumentada. Por meio de marcadores impressos, os alunos podem visualizar os terrenos em RA em qualquer dispositivo com webcam e acesso à internet, com links para as visualizações em RV.

As maquetes construídas no Sketchup têm as superfícies topográficas modeladas por meio da plataforma Digital Globe, possibilitando a criação de curvas de nível, seções planas, planos de taludes para aterros e cortes. As visualizações melhoram a percepção dos alunos das construções feitas em disciplinas que envolvem conceitos de projeção, pois os alunos podem conferir as construções feitas em 2D com a sobreposição das maquetes em 3D. Desta forma, o ensino de formas básicas de 
relevo fica mais interessante, efetivo e dinâmico, pois inclui a simulação da realidade nos desenhos construídos pelos alunos.

$O$ resultado mostra que os ambientes desenvolvidos são ferramentas úteis para uso em sala de aula, pois permitem que os alunos visualizem e manipulem as representações gráficas das maquetes com seus dispositivos ou empregando os óculos de Realidade Virtual para imersão completa na cena. Todos os dispositivos testados pelos alunos das turmas das disciplinas de Projeções Cotadas carregaram os sites sem a ocorrência de erros, mostrando a versatilidade da ferramenta, que funciona em qualquer sistema operacional.

Os ambientes programados podem ser explorados também em aulas de disciplinas de Topografia e Geografia. Todos os elementos podem ser visualizados em RA e RV e os alunos podem movimentar a câmera da cena para encontrarem os melhores pontos de vista das maquetes em RV com funções desenvolvidas para o A-frame para orbitar a câmera em torno dos objetos.

Algumas vantagens da criação de ambientes de RA e RV como páginas da web para uso em sala de aula são a praticidade, baixo custo, ótimo desempenho, a simplicidade de programação e o funcionamento em todos os tipos de smartphones, tablets e notebooks. Com o acesso aos sites em RA e $\mathrm{RV}$, os alunos podem visualizar as maquetes virtuais a qualquer momento e em qualquer lugar de uma forma muito prática, superando restrições que os alunos teriam se tivessem somente acesso às maquetes físicas.

Outra vantagem dos ambientes mostrados neste trabalho é o carregamento quase que imediato dos sites, pois são programados em HTML com referências de bibliotecas de RV desenvolvidas em Java. Os alunos não precisam fazer download de aplicativos e diversos marcadores podem ser usados em uma mesma página de HTML, para criação de materiais didáticos com diversos temas programados em RA e RV. Ambientes similares podem ser usados em outras disciplinas, tais como Geometria, Cálculo Diferencial e Integral, Estatística, Biologia, Química, Engenharia e outras áreas que utilizam representações gráficas em 3D.

\section{Referências}

Abdullah, F., Kassim, M. H. B., \& Sanusi, N. Z. (2017). Go virtual: exploring augmented reality application in representation of steel architectural con- struction for the enhancement of architecture education. Advanced Science Letters, 23(2), 804-808. doi: https://doi.org/10.1166/asl.2017.7449.

A-frame. (2019). A-frame documentation of Virtual Reality. URL: https://aframe.io/docs/0.8.0/introduction. Acesso 20.05.2019.

Akçayır, M., \& Akçayır, G. (2017). Advantages and challenges associated with augmented reality for education: A systematic review of the literature. Educational Research Review, 20, 1-11. doi: https:// doi.org/10.1016/j.edurev.2016.11.002.

Andrade, G., Oliveira, A. C. C. A., \& Diniz, M. C. A. (2018). Geografia e Sandbox: Contribuições da Realidade Aumentada para o Ensino das Formas de Relevo. Congresso sobre tecnologias na Educação, Fortaleza, CE, Brasil, 3, 258-270.

Attardi, S. M., \& Rogers, K. A. (2015). Design and implementation of an online systemic human anatomy course with laboratory. Anatomical Sciences Education, 8(1), 53-62. doi: 10.1002/ase.1465.

Becker, E. L. S., \& Nunes, M. P. (2012). Relevo do Rio Grande do Sul, Brasil, e sua representação em maquete. Revista Percurso, 4(2), 113-132.

Cantos, L. C., Izquierdo, J. L., \& Cantos, E. C. (2016). Interactive multimedia application for teaching and learning in Analytical Geometry. IEEE Latin America Transactions, 14(7), 3461-3466. doi: 10.1109/TLA.2016.7587655.

Carrera, C. C., \& Asensio, L. A. B. (2017). Landscape interpretation with augmented reality and maps to improve spatial orientation skill. Journal of $\mathrm{Ge}$ ography in Higher Education, 41(1), 119-133. doi: 10.1080/03098265.2016.1260530.

Cerra, P. P., Rodríguez, J. G., Álvarez, H. F., \& Parra, B. B. (2018). Combining multimedia and self-assessment CAD tools in an interactive web environment to learn engineering drawing. Interactive Learning Environments, 27(2), 1-14. doi: https://doi. org/10.1080/10494820.2018.1517095.

Digital Globe. (2019). Explore geospatial data in context. Digital Globe. URL: https://www.digitalglobe. com. Acesso 20.05.2019.

Etienne, J. (2017). Efficient Augmented Reality for the Web. URL: https://github.com/jeromeetienne/AR.js. Acesso 20.05.2019.

González, N. A. A. (2017). Development of spatial skills with virtual reality and augmented reality. International Journal on Interactive Design and Manufacturing, 12(1), 133-144. doi: 10.1007/s12008-017-0388-x.

Hinrichs, T. (2019). An Orbit Controls Component for A-Frame VR. URL: https://github.com/tizzle/aframe-orbit-controls-component. Acesso 20.05.2019.

Huleihil, M. (2017). 3D printing technology as innovative tool for math and geometry teaching applications. IOP Conference Series: Materials Science and Engineering, 164(1), 1-7. doi: https://doi. org/10.1088/1757-899X/164/1/012023. 
Irwansyah, F. S., Yusuf, Y. M., Farida, I., \& Ramdhani, M. A. (2018). Augmented Reality (AR) Technology on The Android Operating System in Chemistry Learning. IOP Conference Series: Materials Science and Engineering, 288(1), 233-237. doi: 10.1088/1757-899X/288/1/012068.

Ishii, H. (2010). Augmented Reality: Fundamentals and Nuclear Related Applications. International Journal of Nuclear Safety and Simulation, 1(4), 316-327.

Kirner, C., \& Kirner, T. G. (2011). Development of an interactive artifact for cognitive rehabilitation based on augmented reality. International Conference on Virtual Rehabilitation (ICVR'11), Zurich, Switzerland, 1-7. doi: 10.1109/ICVR.2011.5971837.

McMenamin, P. G., Quayle, M. R., McHenry, C. R., \& Adams, J. W. (2014). The production of anatomical teaching resources using three-dimensional (3D) printing technology. Anatomical Sciences Education, 7(6), 479-486. doi: 10.1002/ase.1475.

Moro, C., Stromberga, Z., Raikos, A., \& Stirling, A. (2017). The effectiveness of virtual and augmented reality in health sciences and medical anatomy. Anatomical sciences education, 10(6), 549-559. doi: 10.1002/ase.1696.

Munoz-Cristóbal, J. A., Gallego-Lema, V., Arribas-Cubero H. F., Asensio-Pérez, J. I., \& Martínez-Monés, A. (2018). Game of Blazons: Helping Teachers Conduct Learning Situations That Integrate Web Tools and Multiple Types of Augmented Reality. IEEE Transactions on learning technologies, 11(4), 506-519. doi: https://doi.org/10.1109/ TLT.2018.2808491.

Murphy, W. (2019). All-in-one natural hand controller, pointer, and gaze interaction library for A-Frame. URL: https://github.com/wmurphyrd/aframe-super-hands-component. Acesso 20.05.2019.
Oliveira, J. R. (2018). Visualização de conteúdos multimédia num ambiente de realidade virtual (Dissertação de Mestrado). Faculdade de Engenharia da Universidade do Porto, Porto, Portugal.

Santos, E. T., Mafalda, R., Kawano, A., Cheng, L. Y., Petreche, J. R. D., Leite, B. C. C., Ferreira, S. L., \& Cardoso, L. R. A. (2003). Da geometria cotada ao modelamento 3D: projeto didático. Simpósio Nacional de Geometria Descritiva e Desenho Técnico e International Conference on Graphics Engineering for Arts and Design, Santa Cruz do Sul, RS, Brasil, 16, 1-7.

Santos, R. S., Santos, R. S., Alencar, C. M. S., \& Macedo, C. M. S. (2018). Realidade Aumentada no Processo de Ensino-Aprendizagem da Topografia em Projeto de Engenharia Civil. Congresso sobre tecnologias na Educação, Fortaleza, CE, Brasil, 3, 582-589.

Sena, C. C. R. G., \& Carmo, W. R. (2018). Cartografia Tátil: o papel das tecnologias na Educação Inclusiva. Boletim Paulista de Geografia, 99, 102-123.

Serrano, F. (2017). A-Frame teleport controls component. URL: https://github.com/fernandojsg/ aframe-teleport-controls. Acesso 20.05.2019.

Sketchup. (2019). 3D Design Software. URL: https:// www.sketchup.com. Acesso 20.05.2019.

Silva, C. M., \& Ferreira, G. C. (2008). Produção de material didático: jogo das curvas de nível. Boletim Goiano de Geografia, 28, 157-170. doi: 10.5216/bgg. v28i2.5736.

Sousa, R. R. (2014). Oficina de maquete de relevo: um recurso didático. Terrae Didatica, 10(1), 22-28. doi: https://doi.org/10.20396/td.v10i1.8637385.

Souza, W. O., Espíndola, G. M., Pereira, A. R. A., \& Sá, L. A. C. M. (2016). A realidade aumentada na apresentação de produtos cartográficos. Boletim de Ciências Geodésicas, 22(4), 790-806. doi: 10.1590/ S1982-21702016000400045. 